Delft University of Technology

\title{
A Linear Array of Skewed Dipoles With Asymmetric Radiation Pattern for Angular Filtering
}

Yepes, C.; Gandini, E.; Monni, S.; Neto, A.; Vliet, F. E. van; Cavallo, D.

DOI

10.1109/LAWP.2020.2971347

Publication date

2020

Document Version

Accepted author manuscript

Published in

IEEE Antennas and Wireless Propagation Letters

\section{Citation (APA)}

Yepes, C., Gandini, E., Monni, S., Neto, A., Vliet, F. E. V., \& Cavallo, D. (2020). A Linear Array of Skewed Dipoles With Asymmetric Radiation Pattern for Angular Filtering. IEEE Antennas and Wireless Propagation Letters, 19(3), 408-412. [8985266]. https://doi.org/10.1109/LAWP.2020.2971347

\section{Important note}

To cite this publication, please use the final published version (if applicable).

Please check the document version above.

\section{Copyright}

Other than for strictly personal use, it is not permitted to download, forward or distribute the text or part of it, without the consent of the author(s) and/or copyright holder(s), unless the work is under an open content license such as Creative Commons.

\section{Takedown policy}

Please contact us and provide details if you believe this document breaches copyrights.

We will remove access to the work immediately and investigate your claim. 


\title{
A Linear Array of Skewed Dipoles with Asymmetric Radiation Pattern for Angular Filtering
}

\author{
Cristina Yepes, Student Member, IEEE, Erio Gandini, Member, IEEE, Stefania Monni, Senior Member, IEEE, \\ Andrea Neto, Fellow, IEEE, Frank E. van Vliet, Senior Member, IEEE, Daniele Cavallo, Senior Member, IEEE
}

\begin{abstract}
We present a design of a linear array of tilted dipoles to achieve radiation patterns with asymmetric angular filtering characteristics. To realize the asymmetric radiation, the dipole elements are spaced by a distance larger than half a wavelength, thus allowing for grating lobes to occur in the visible region. Moreover, the dipoles are loaded with artificial dielectrics to increase the front-to-back ratio and consequently to enable higher gain in certain desired angular regions. Based on the design, a linear array with 10 elements is manufactured and tested. The measured results show the ability of such an array to achieve stable gain from broadside up to 90 degrees scanning, while implementing a stop-band angular filter for negative scanning angles.
\end{abstract}

Index Terms-Angular filtering, antenna arrays, pattern asymmetry, tilted dipole array.

\section{INTRODUCTION}

$\mathbf{M}$ ODERN antenna arrays for radar and communication applications often operate in environments where a multitude of sensors and radiating systems need to co-exist. To avoid interference between different antenna systems, the implementation of angular filtering functions can be beneficial. Moreover, in some of the mentioned applications, it is sometimes not required for the antennas to exhibit a field of view that is symmetric with respect to broadside, but rather a gain profile that is stable over a certain desired angular region. For instance, to improve the system capacity of basestations antennas, a down-tilt of the radiation towards the ground was employed to increase the signal reception from the mobile device [1], [2]. In satellite communications, tilting the embedded pattern of the individual elements of an antenna array was implemented to achieve a gain enhancement for large scan angles within a limited azimuth region [3], [4]. The entire field of view was then covered by mechanical rotation of the array along its axis.

Manuscript received Month DD, YYYY; revised Month DD YYYY. First published Month DD, YYYY; current version published Month DD, YYYY This work was supported by by the TNO Ph.D. funding. (Corresponding author: Cristina Yepes.)

C. Yepes, A. Neto, and D. Cavallo are with the Microelectronics Department of the Electrical Engineering, Mathematics and Computer Science Faculty, Delft University of Technology, 2628 CD Delft, The Netherlands (e-mail: c.yepesjulia@tudelft.nl).

C. Yepes, E. Gandini, S. Monni, and F.E. van Vliet are with the Department of Radar Technology, TNO, 2597 AK The Hague, The Netherlands. F. van Vliet is also with the Center for Array Technology, University of Twente, 7522 NB Enschede, The Netherlands.

Color versions of one or more of the figures in this paper are available online at http://ieeexplore.ieee.org.

Digital Object Identifier XX.XXXX/TAP.XXXX.XXXXXXXX

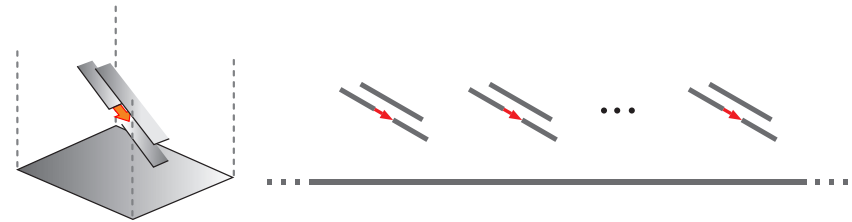

(a)

(b)

Fig. 1. Array of titled stacked dipole elements, in the presence of an infinite ground plane: (a) 3D-view of the unit cell and (b) side view.

Angular filters have been realized mainly with frequency selective surfaces or leaky wave structures [5]-[7], with the aim of increasing the directivity and reducing the levels of the side lobes or grating lobes. In [8], [9], rectilinear leaky-wave antennas were synthesized to realized radiation patterns with angular selectivity and asymmetry properties. However, with this concept, the entire array radiates a broad beam with an angular passband filtering function, precluding the possibility to generate highly directive beams that can be scanned within the field of view of interest.

In this work, the proposed approach is to realize an antenna array where the single element exhibits a radiation pattern with angular selectivity, asymmetric with respect to broadside. The resulting active element pattern will filter the array factor of the entire array while scanning, providing high gain only in the desired field of view. Recently in [10] we showed that one effective way to achieve asymmetric active element patterns is by enhancing the directivity of the element (e.g. with a dipole loaded by a parasitic strip as director) and by rotating the array element around their center, as shown in Fig. 1. In [10], the fundamental radiation properties of arrays of stacked dipoles were investigated, based on a spectral method of moments (MoM) analysis. The results are consistent with the general theory of arrays with asymmetric elements given in [11], [12]. Based on the findings provided in [10], we propose here the design of a linear array of tilted dipole elements to achieve asymmetric radiation characteristics. The dipoles are loaded with artificial dielectrics to increase the gain in certain desired angular regions. An implementation of the array based on printed circuit technology (PCB) is proposed, as well as the design of the feed structure. To validate the design, a prototype linear array with 10 elements is manufactured and tested and the measured results are reported.

\section{Unit Cell Design}

In [10], the guidelines to design an array with asymmetric radiation properties were discussed. The main unit cell under 


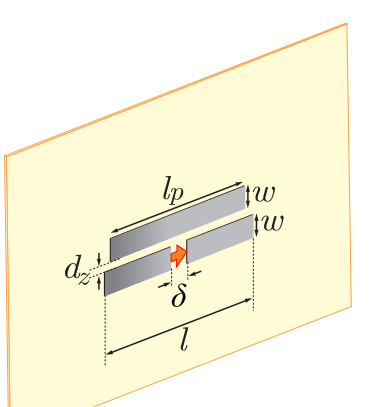

(a)

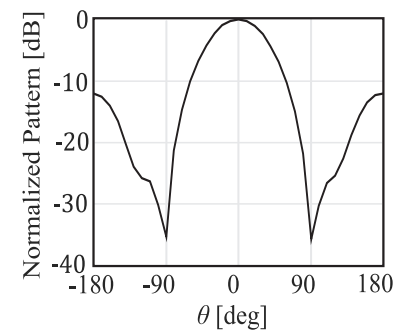

(b)

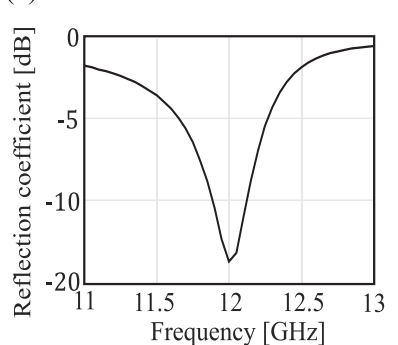

(c)
Fig. 2. (a) Single element of a stacked dipole printed on a PCB, (b) E-plane pattern and (c) reflection coefficient of the single element described in (a).

analysis consisted in an idealized element with infinitely thin flat strip dipoles, fed with a delta-gap excitation, as shown in Fig. 1(a). In this section, we propose a realistic implementation of the element, based on PCB technology.

\section{A. Single Dipole Element with Parasitic Loading}

To enable a simple realization of the flat strip dipole element, we first consider an equivalent isolated element where the active dipole and the parasitic strip are printed on a vertically oriented thin dielectric slab. Figure 2(a) shows the considered structure with the characteristic geometrical parameters. The dimensions of the elements are chosen as: $l=0.41 \lambda_{0}, l_{p}=0.37 \lambda_{0}, w=0.05 \lambda_{0}, \delta=0.01 \lambda_{0}$ and $d_{z}=0.7 \lambda_{0}$, where $\lambda_{0}$ is the wavelength at $12 \mathrm{GHz}$. The dielectric slab selected for the design is Rogers RO4530 TM with relative dielectric constant $\varepsilon_{r}=3.66$ and thickness $t=101 \mu \mathrm{m}$. The dimensions are optimized so that the directivity of the element is maximized. The simulated $E$ plane pattern of a the single element in free space is reported in Fig. 2(b), at $12 \mathrm{GHz}$, exhibiting a front-to-back ratio of $12 \mathrm{~dB}$. Figure 2(c) depicts the simulated reflection coefficient of the element, showing a rather narrow bandwidth of only $2 \%$ centered at $12 \mathrm{GHz}$, normalized to a low impedance of $10 \Omega$.

To enhance the front-to-back ratio of the element as well as the bandwidth, a solution consisting of a tilted dipole element loaded with 3 layers of artificial dielectric (AD) is proposed in Fig. 3(a). The AD consists of sub-wavelength metal strips on the same plane of the dipole. This element is also printed on a vertical PCB with a dielectric with the same relative permittivity and thickness as for the stacked dipole solution $\left(\varepsilon_{r}=3.66, t=101 \mu \mathrm{m}\right)$. The dimensions, reported in Table I, were tuned to enhance the directivity of the element. The

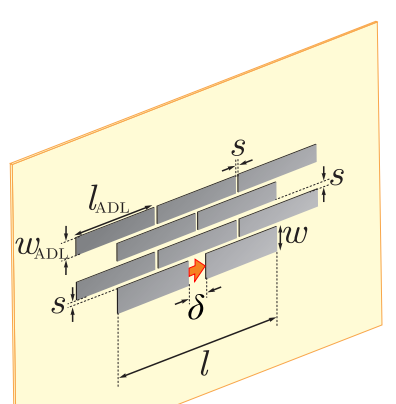

(a)

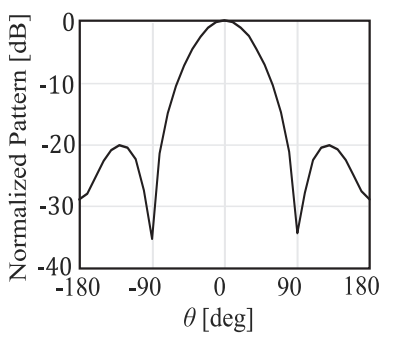

(b)

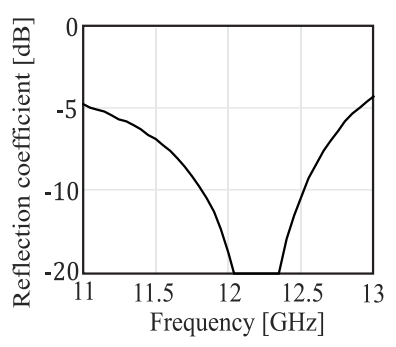

(c)
Fig. 3. (a) Single element of a dipole loaded with artificial dielectric layer printed on a $\mathrm{PCB}$, and its (b) single element $E$-plane pattern and (c) reflection coefficient.

TABLE I

Geometrical Parameter of Element in Fig. 3, with Respect to THE WAVELENGTH $\lambda_{0}$ AT $12 \mathrm{GHz}$.

\begin{tabular}{cccccc}
$l$ & $w$ & $\delta$ & $l_{\mathrm{ADL}}$ & $w_{\mathrm{ADL}}$ & $s$ \\
\hline $0.434 \lambda_{0}$ & $0.05 \lambda_{0}$ & $0.05 \lambda_{0}$ & $0.212 \lambda_{0}$ & $0.0336 \lambda_{0}$ & $0.01 \lambda_{0}$ \\
\hline
\end{tabular}

single element $E$-plane pattern at $12 \mathrm{GHz}$ and the reflection coefficient are shown in Fig. 3(b) and (c), respectively. The dipole loaded with artificial dielectric has a back lobe with $-20 \mathrm{~dB}$ level and a bandwidth of $6 \%$, with respect to the normalization impedance of $30 \Omega$. The gain difference between this configuration and a regular dipole is $4.4 \mathrm{~dB}$.

\section{B. Infinite Array of Dipoles Loaded with Artificial Dielectrics}

We now consider the element in Fig. 3(a), tilted by an angle $\alpha$ and embedded in an infinite linear array environment, in the presence of a backing reflector. The resulting unit cell is depicted in Fig. 4(a). The distance between the center of the dipole and the infinite ground plane is $h_{\mathrm{gp}}=0.25 \lambda_{0}$ and the total height of the dielectric is $h=0.7 \lambda_{0}$.

To describe the angular filtering function, let us imagine to divide the angular region between $-90^{\circ}$ and $90^{\circ}$ in two portions, one desired, where high gain is wished for, and one undesired, where gain suppression is targeted (Fig. 4(b)). If the angle bounding these two regions is referred to as $\theta_{\text {drop }}$, from the study done in [10], we can derive an optimal inter-element spacing and inclination angle of the elements to achieve a desired $\theta_{\text {drop }}$. For example, the choice of $\theta_{\text {drop }}=-20^{\circ}$ results in an optimal period of $d_{x}=0.75 \lambda_{0}$ and an optimal inclination angle of $\alpha=40^{\circ}$. Figure 4(b) reports the simulated active element pattern in the $E$-plane at $12 \mathrm{GHz}$. The radiation pattern shows a clear drop in gain in the range $-90^{\circ}<\theta<-30^{\circ}$, a roll-off region centered around the target value $\theta_{\text {drop }}=-20^{\circ}$, 


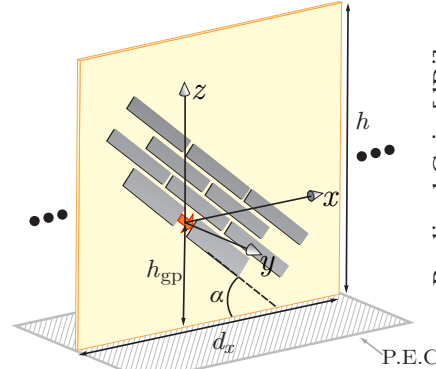

(a)

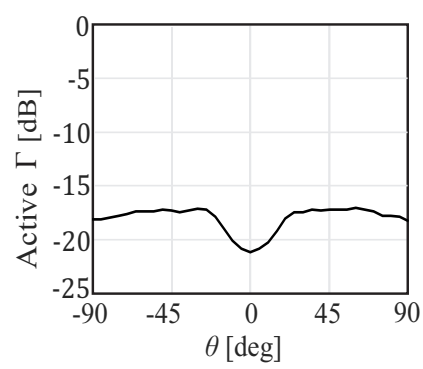

(c)

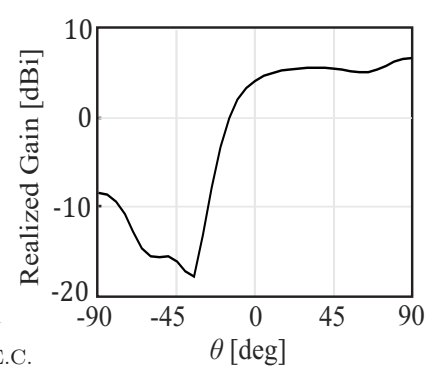

(b)

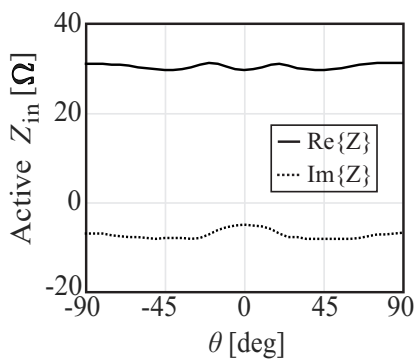

(d)
Fig. 4. (a) Infinite one-dimensional array unit cell of a tilted dipole with artificial dielectric loading, in the presence of a backing reflector; (b) E-plane realized gain active element pattern, (c) active reflection coefficient and (d) active input impedance, versus scan angle.

and and almost constant value for angles between $0^{\circ}$ and $90^{\circ}$. The active reflection coefficient at $12 \mathrm{GHz}$ and the active input impedance are shown in Fig. 4(c) and (d), respectively, as a function of the scan angle. It can be noted that the impedance is almost constant with scan angle, which is a consequence of the low mutual coupling between the elements. Indeed, the large interelement spacing $\left(0.75 \lambda_{0}\right)$ yields low coupling, thus the active input impedance is close to the passive input impedance and hardly dependent on the scan angle.

\section{Feed Design}

The last step of the unit cell design consists in replacing the ideal delta-gap feed with a realistic feeding structure. We employ a microstrip feeding line with a Marchand balun to avoid the excitation of common mode currents. A two quarter-wave section transmission line is used to transform the $30 \Omega$ impedance at the antenna terminals up to $50 \Omega$ at the coaxial connector input. For the sake of simple realization, the horizontal ground plane is replaced by a vertical ground plane printed on the same PCB as the dipole and the feeding lines (see Fig. 5(a)). The parameters of the feed structure are illustrated in Fig. 5(b) and Fig. 5(c) and their values can be found in Table II.

Figure 6(a) shows the simulated active element pattern, comparing directivity and realized gain. The simulation includes the feeding lines and the connector and accounts for the material losses, both metal and dielectric. The difference between directivity and gain is about $0.8 \mathrm{~dB}$, which corresponds to a simulated efficiency of $83 \%$. We can observe that gain levels in the undesired region are about $-15 \mathrm{~dB}$ below the maximum gain, similarly to the previous results for the ideal feed. Figure 6(b) reports the active input impedance at

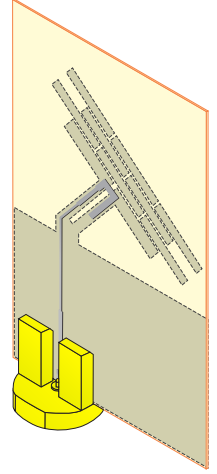

(a)

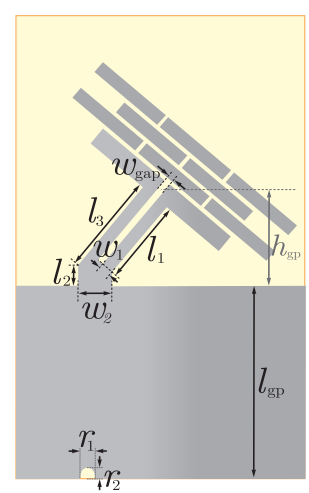

(b)

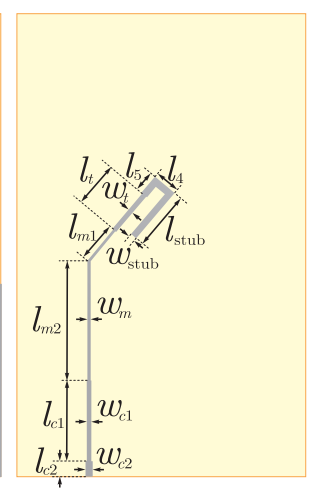

(c)
Fig. 5. (a) 3D view of the array element with an SMA connector, (b) front and (c) back of the unit cell design with the radiating element, the feeding lines with a Marchand balun and a vertical ground plane.

TABLE II

Geometrical Parameter of the Feed Structure (IN mm)

\begin{tabular}{cccccccc}
$w_{\text {gap }}$ & $l_{1}$ & $l_{2}$ & $l_{3}$ & $l_{\mathrm{gp}}$ & $w_{1}$ & $w_{2}$ & $l_{\text {stub }}$ \\
\hline 0.4 & 6.55 & 1.39 & 5.5 & 12.5 & 0.8 & 2.21 & 3.85 \\
\hline \multirow{2}{*}{$l_{t}$} & $l_{m 1}$ & $l_{m 2}$ & $l_{4}$ & $l_{5}$ & $l_{c 1}$ & $l_{c 2}$ & $w_{\text {stub }}$ \\
\hline 3 & 2.6 & 14.04 & 1.65 & 1.35 & 5.25 & 1 & 0.45 \\
\hline \multicolumn{1}{c}{$w_{t}$} & $w_{m}$ & $w_{c 1}$ & $w_{c 2}$ & $r_{1}$ & $r_{2}$ & \\
& 0.3 & 0.18 & 0.3 & 0.6 & 1.02 & 0.75 &
\end{tabular}

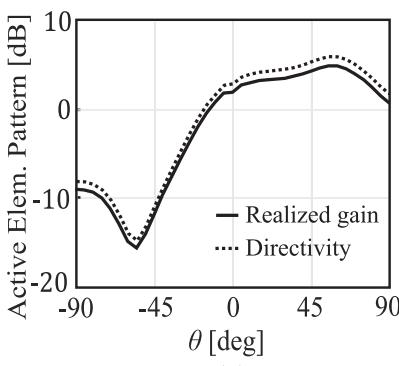

(a)

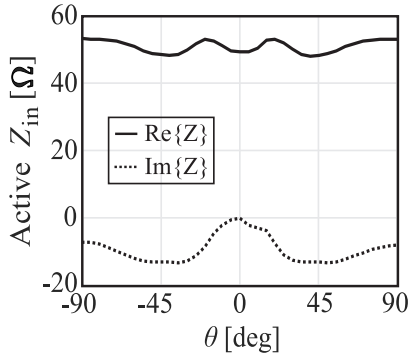

(b)
Fig. 6. (a) E-plane radiation pattern and (b) input impedance while varying the scanning angle of the unit cell shown in Fig. 5(a).

$12 \mathrm{GHz}$, versus the scan angle. The impedance is well matched to $50 \Omega$ and marginally varying with the scan angle.

\section{ARRAy PRototype}

Based on the unit cell design presented in the previous section, a finite linear array comprising 10 elements was manufactured (see Fig. 7). A subset of the array S-parameters was measured, i.e. the coupling coefficients of the elements with index 1,5 and 10 with all the others. This allows to compute the active reflection coefficient of one central element (element 5) and the two edge elements (1 and 10) for different scan angles. The results are shown in Fig. 8. Simulations of the finite array are also reported in the same figure for comparison. The elements show a matching bandwidth of about $10 \%$, which is almost constant when scanning.

The measured gain active element patterns in the $E$-plane are reported in Fig. 9(a), for a few array elements, at a frequency of $12 \mathrm{GHz}$. Because of truncation effects in the finite 


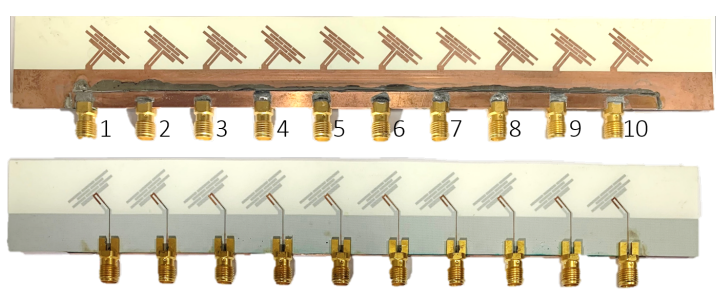

Fig. 7. Photo of the front and back of the 10-element linear array prototype.
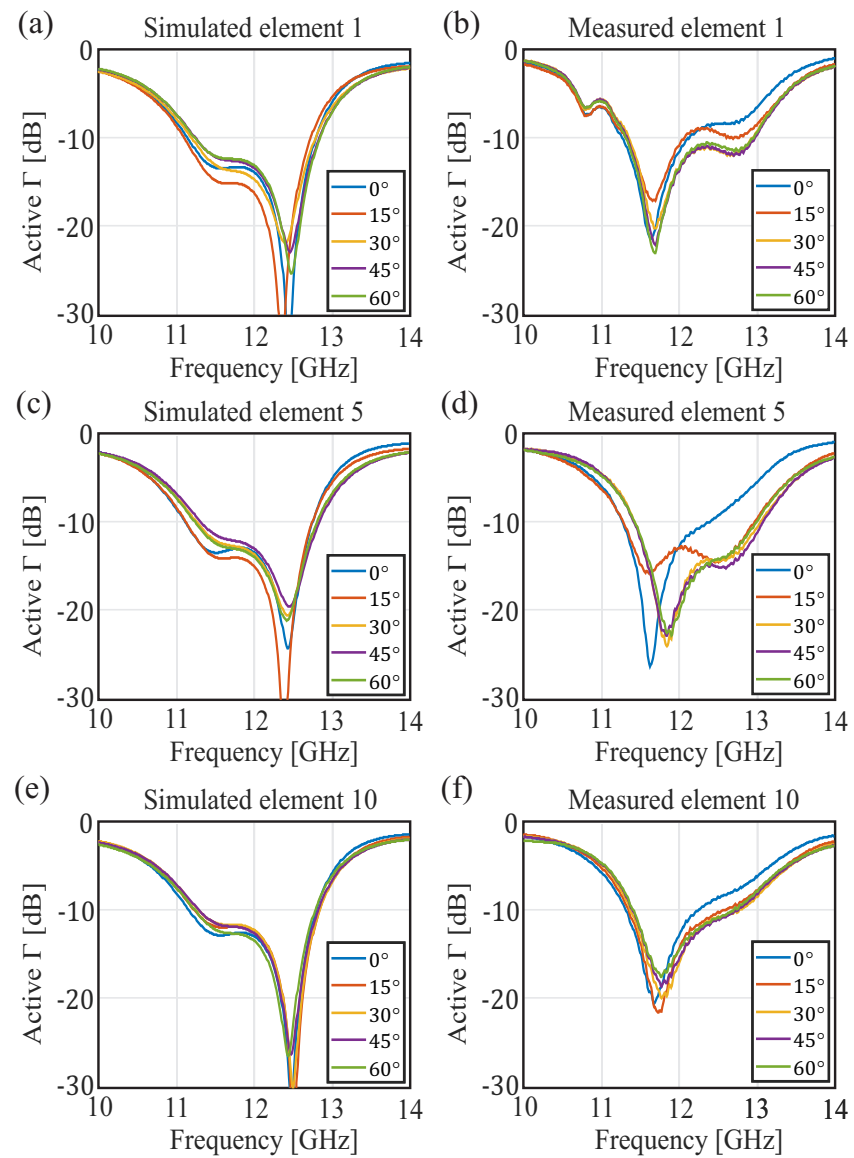

Fig. 8. Simulated and measured active reflection coefficient for (a) and (b) element 1, (c) and (d) element 5, and (e) and (f) element 10.

array, the gain patterns differ for each element. For example, element 1 displays a lower level side-lobe pointing at $-90^{\circ}$, compared to other elements. All measured radiation patterns closely resemble the active element pattern simulated with the infinite array approximation, also shown in Fig. 9(a). Larger discrepancies occur at angles close to $\pm 90^{\circ}$. This is due to some reflections from the plastic frame holding the antenna, used for the measurements.

The $H$-plane gain pattern of the central element of the array is shown in Fig. 9(b), comparing simulation with measurements. Also the cross-polarized component is shown in the figure. In fact, in the $E$-plane, the cross-pol is not affected by the tilt of the dipoles nor by the feeding structure, since both the planar and vertical components of the current radiate co-polar fields. However, in the $H$-plane, the cross-polar component increases for angles away from broadside, due to

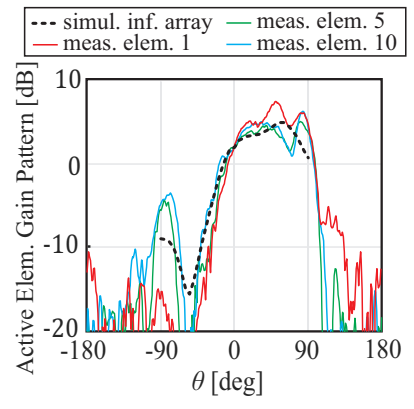

(a)

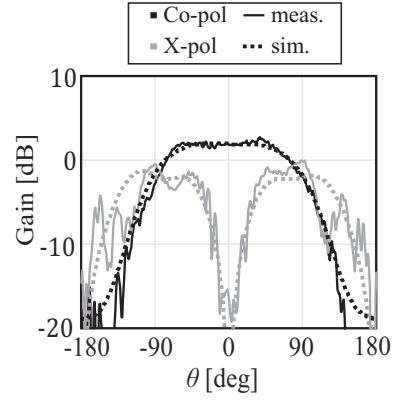

(b)
Fig. 9. (a) Active element pattern (gain) of three elements of the array, compared with infinite array simulations. (b) Co-polar and cross-polar $H$-plane gain pattern of the element 5 of the array, compared with simulations.
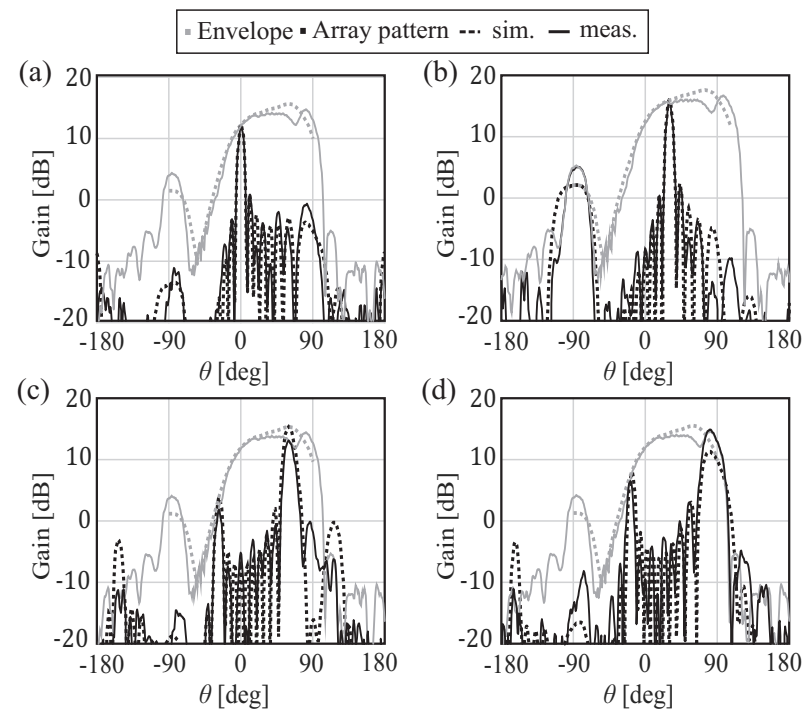

Fig. 10. Measured and simulated array gain patterns for different scan angles: Scan to (a) $\theta=0^{\circ}$, (b) $\theta=20^{\circ}$, (c) $\theta=60^{\circ}$, (d) $\theta=90^{\circ}$.

the tilting and the radiating currents.

By summing the measured active elements patterns with the proper phases, we can also estimate the total array patterns. The resulting gain patterns are shown in Fig. 10, compared with simulations. When scanning to $20^{\circ}$, the grating lobe entering in the visible region can be observed at $-90^{\circ}$, but its level remains $-10 \mathrm{~dB}$ below the maximum, because it is weighted by the filtering function of the element pattern.

\section{CONCLUSIONS}

We presented the design of a linear array of dipoles with asymmetric radiation properties. The array elements consist of tilted dipoles with parasitic artificial dielectric layers to enhance their gain. The pattern of such an element can be designed to exhibit a high-gain desired region for angles in the range $\theta_{\text {drop }}<\theta<90^{\circ}$ and a low-gain undesired region for $-90^{\circ}<\theta<\theta_{\text {drop }}$. The angle $\theta_{\text {drop }}$ can be controlled by the inter-element distance, while the difference in gain between the desired and undesired regions can be optimized by the tilt angle of the elements. 
A prototype array based on this concept was presented, showing a good comparison between simulated and measured characteristics.

\section{REFERENCES}

[1] D. Sugimura, M. Arai, K. Sakaguchi, K. Araki and T. Sotoyama, "A study on beam tilt angle of base station antennas for Base Station Cooperation systems," IEEE 22nd Int. Symp. Personal, Indoor and Mobile Radio Communications, Toronto, Canada, 2011, pp. 2374-2378.

[2] I. Kim and Y. Rahmat-Samii, "Electromagnetic band gap-dipole sub-array antennas creating an enhanced tilted beams for future base station," IET Microw., Antennas Propag., vol. 9, no. 4, pp. 319-327, Mar. 2015.

[3] F. Tiezzi, D. Llorens, C. Dominguez, and M. Fajardo, "A compact Kuband transmit/receive low-profile antenna for broadband mobile satellite communications," Proceedings of the Fourth European Conference on Antennas and Propagation (EuCAP), pp. 1-4 Barcelona, Spain, 2010.

[4] J. Biosca, D. Llorens, and M. C. Viganó, "Side-lobe reduction with overlapped beam-forming network for Ku-band hybrid antenna array," 10th Eur. Conf. Antennas Propag., pp. 1-5, Davos, 2016.

[5] P. Franchi and R. Mailloux, "Theoretical and experimental study of metal grid angular filters for sidelobe suppression," IEEE Trans. Antennas Propag., vol. 31, no. 3, pp. 445-450, May 1983.

[6] Y. J. Lee, S. H. Jeong, W. S. Park, J. S. Yun, and S. I. Jeon, "Multilayer spatial angular filter with airgap tuners to suppress grating lobes of $4 \times 1$ array antenna," Electronics Lett., vol. 39, no. 1, pp. 15-17, Jan. 2003.

[7] D. Blanco, N. Llombart, and E. Rajo-Iglesias, "On the use of leaky wave phased arrays for the reduction of the grating lobe level," IEEE Trans. Antennas Propag., vol. 62, no. 4, pp. 1789-1795, Apr.2014.

[8] P. Burghignoli, F. Frezza, A. Galli, and G. Schettini, "Synthesis of broadbeam patterns through leaky-wave antennas with rectilinear geometry," IEEE Antennas Wireless Propag. Lett., vol. 2, pp. 136-139, 2003.

[9] A. J. Martinez-Ros, J. L. Gómez-Tornero and G. Goussetis, "Multifunctional angular bandpass filter SIW leaky-wave antenna," IEEE Antennas Wireless Propag. Lett., vol. 16, pp. 936-939, 2017.

[10] C. Yepes, E. Gandini, S. Monni, A. Neto, F. E. van Vliet, and D. Cavallo, "Analysis of tilted dipole arrays: Impedance and radiation properties," IEEE Trans. Antennas Propag., vol. 68, no. 1, pp. 254-265, Jan. 2020.

[11] A. K. Bhattacharyya, "Active element pattern symmetry for asymmetrical element arrays," IEEE Antennas Wireless Propag. Lett., vol. 6, pp. 275-278, 2007.

[12] H. Steyskal, "On the merit of asymmetric phased array elements," IEEE Trans. Antennas Propag., vol. 61, no. 7, pp. 3519-3524, Jul. 2013. 